Article

\title{
Sonogashira Reaction of Aryl and Heteroaryl Halides with Terminal Alkynes Catalyzed by a Highly Efficient and Recyclable Nanosized MCM-41 Anchored Palladium Bipyridyl Complex
}

\author{
Bo-Nan Lin ${ }^{1}$, Shao-Hsien Huang ${ }^{1}$, Wei-Yi Wu ${ }^{1}$, Chung-Yuan Mou ${ }^{2}$ and Fu-Yu Tsai ${ }^{1, *}$ \\ 1 Institute of Organic and Polymeric Materials, National Taipei University of Technology, Taipei \\ 106, Taiwan \\ 2 Department of Chemistry, National Taiwan University, Taipei 106, Taiwan \\ * Author to whom correspondence should be addressed; E-Mail: fuyutsai@ntut.edu.tw; \\ Fax: +886-2-2731-7174.
}

Received: 11 November 2010; in revised form: 6 December 2010 / Accepted: 9 December 2010 / Published: 10 December 2010

\begin{abstract}
A heterogeneous catalyst, nanosized MCM-41-Pd, was used to catalyze the Sonogashira coupling of aryl and heteroaryl halides with terminal alkynes in the presence of $\mathrm{CuI}$ and triphenylphosphine. The coupling products were obtained in high yields using low Pd loadings to $0.01 \mathrm{~mol} \%$, and the nanosized MCM-41-Pd catalyst was recovered by centrifugation of the reaction solution and re-used in further runs without significant loss of reactivity.
\end{abstract}

Keywords: Sonogashira reaction; mesoporous silica; palladium complex; recyclable catalyst; heterogeneous catalysis

\section{Introduction}

The reaction of aryl halides or vinyl halides with terminal alkynes catalyzed by a $\mathrm{Pd}(\mathrm{II}) / \mathrm{Cu}(\mathrm{I})$ system is known as the Sonogashira coupling, and is one of the most powerful methods for the straightforward construction of $s p^{2}-s p$ carbon-carbon bonds in synthetic chemistry [1-7]. This methodology has been widely applied to prepare biologically-active molecules [8-13], natural 
products [14-17], conducting polymers/engineering materials [18,19], and macrocycles with acetylene links [20,21].

The Sonogashira reaction is, in general, carried out in a homogeneous phase [22], and therefore the recovery of expensive palladium complexes, facile separation of catalysts and products, and industrial application are major aims for the benefit of both economy and the environment. For these reasons, heterogenization of the homogeneous Sonogashira reaction has become an aim of great interest to chemists in recent years. Choudary and co-workers described a layered double hydroxide-supported nanopalladium catalyst for the coupling of aryl chlorides and phenylacetylene [23], and $\mathrm{Pd} / \mathrm{C}$ has been used to catalyze the Sonagashira reaction of aryl halides with acetylenes [24-30], while PVPsupported nanoparticle palladium metal can be employed for the coupling of aryl iodides and bromides with terminal alkynes [31]. Djakovitch and co-workers reported that microporous [Pd-Cu]/NaY [32], $\left[\mathrm{Pd}\left(\mathrm{NH}_{3}\right)_{4}\right]^{2+} /\left(\mathrm{NH}_{4}\right) \mathrm{Y}[33]$, and $\left[\mathrm{Pd}\left(\mathrm{NH}_{3}\right)_{4}\right]^{2+} / \mathrm{NaY}$ [34] systems can be applied in the Sonogashira reaction using 1-2 mol\% of the Pd catalyst, and palladium can be also supported by silica in order to create a recyclable catalyst for use in the Sonogashira reaction [35].

Mesoporous silica is becoming more and more widely used as a solid support owing to its welldefined structure, uniform pore size, high surface area, and large number of silanol groups for the grafting of metal complexes [36-44]. Djakovitch's group prepared a mesoporous [Pd]/SBA-15 catalyst to demonstrate that larger aryl halides such as bromoanthacene can be active in this catalytic system, whereas the microporous support $\left[\mathrm{Pd}\left(\mathrm{NH}_{3}\right)_{4}\right]^{2+} / \mathrm{NaY}$ is inactive [45]. Cai and co-workers employed MCM-41-supported sulfur palladium [46], bidentate phosphine palladium [47], and thioether palladium [48] systems to catalyze the coupling of aryl iodides and terminal alkynes after reduction of the catalyst. Although most known heterogeneous catalysts have been demonstrated to be able to be recycled for use in further runs, the use of catalytic amounts of $0.2-5 \mathrm{~mol} \%$ of $\mathrm{Pd}$ for the Sonagashira reaction is still too high for a single batch reaction when compared with homogeneous catalysts [49-54]. We have recently prepared a nanosized MCM-41 grafted palladium bipyridyl complex, NSMCM-41-Pd (Figure 1), as a highly efficient and recyclable catalyst for the Mizoroki-Heck reaction [55], Kumada-Tamao-Corriu reaction [56], ketone formation [57], and ynone formation [58], which require a very low catalyst loading for a single batch reaction.

Figure 1. NS-MCM-41-Pd.




The major advantage of this catalyst is that the short and highly-connective wormhole-like channels of nanosized MCM-41 lead to the easy exchange of reactants, salts and products throughout the nanochannels, avoiding saturation of activity. In this paper, we report the use of nanosized MCM-41Pd to catalyze the coupling of aryl and heteroaryl halides with phenylacetylene and alkynols with high efficiency under Sonogashira reaction conditions using a catalyst loading as low as $0.01 \mathrm{~mol} \%$, with the ability to recycle the catalyst for further use (Scheme 1).

Scheme 1. NS-MCM-41-Pd-catalyzed Sonogashira reaction.

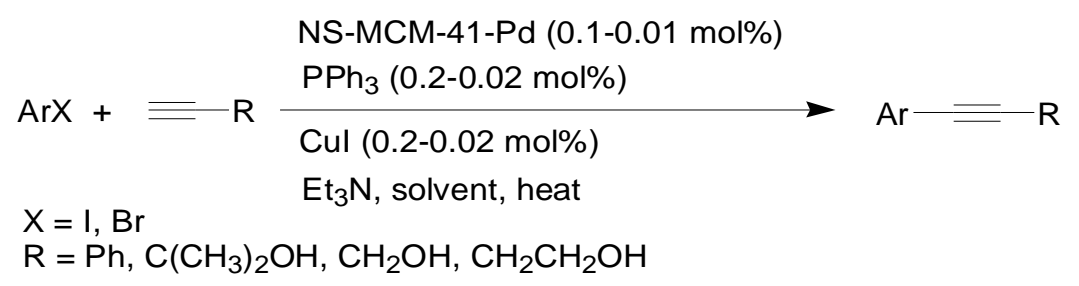

\section{Results and Discussion}

\subsection{Optimization of reaction conditions for the Sonogashira reaction catalyzed by NS-MCM-41-Pd}

The procedure for the synthesis of the catalyst, NS-MCM-41-Pd, was presented in our previous reports. After the grafting of the palladium bipyridyl complex onto NS-MCM-41, the surface area and pore diameter decreased from $705 \mathrm{~m}^{2} / \mathrm{g}$ and $2.5 \mathrm{~nm}$ to $588 \mathrm{~m}^{2} / \mathrm{g}$ and $2.3 \mathrm{~nm}$, respectively, and the amount of Pd complex anchored to the wall of NS-MCM-41 was quantified to be $0.15 \mathrm{mmol} / \mathrm{g}$ by ICPMASS analysis. In order to optimize the conditions for this prepared heterogeneous catalyst, the solvent effect was first examined using iodobenzene (1a) and phenylacetylene (2a) as representative reactants. Reactions were carried out in the presence of $0.1 \mathrm{~mol} \%$ catalyst, $0.2 \mathrm{~mol} \% \mathrm{CuI}, 0.2 \mathrm{~mol} \%$ $\mathrm{PPh}_{3}$, and $\mathrm{Et}_{3} \mathrm{~N}$ at $50{ }^{\circ} \mathrm{C}$ under $\mathrm{N}_{2}$ for $3 \mathrm{~h}$. The results are summarized in Table 1, and it was found that $\mathrm{Et}_{3} \mathrm{~N}$ was the best solvent for this reaction (Table 1, Entry 1).

Table 1. NS-MCM-41-Pd-catalyzed Sonogashira coupling reaction of iodobenzene 1a with phenylacetylene 2a. ${ }^{\text {a }}$

\begin{tabular}{lllllll}
\hline Entry & Pd (mol\%) & CuI (mol\%) & PPh $_{\mathbf{3}} \mathbf{( m o l \% )}$ & Solvent & Base & Yield (\%) \\
\hline 1 & 0.1 & 0.2 & 0.2 & $\mathrm{Et}_{3} \mathrm{~N}$ & $\mathrm{Et}_{3} \mathrm{~N}$ & 97 \\
2 & 0.1 & 0.2 & 0.2 & Toluene & $\mathrm{Et}_{3} \mathrm{~N}^{\mathrm{c}}$ & 60 \\
3 & 0.1 & 0.2 & 0.2 & $\mathrm{DMF}$ & $\mathrm{Et}_{3} \mathrm{~N}^{\mathrm{c}}$ & 34 \\
4 & 0.1 & 0.2 & 0.2 & $\mathrm{DMSO}$ & $\mathrm{Et}_{3} \mathrm{~N}^{\mathrm{c}}$ & 26 \\
5 & 0.1 & 0.2 & 0.2 & $\mathrm{NMP}$ & $\mathrm{Et}_{3} \mathrm{~N}^{\mathrm{c}}$ & 5 \\
6 & 0.1 & 0.2 & 0 & $\mathrm{Et}_{3} \mathrm{~N}$ & $\mathrm{Et}_{3} \mathrm{~N}$ & 38 \\
7 & 0.1 & 0 & 0.2 & $\mathrm{Et}_{3} \mathrm{~N}$ & $\mathrm{Et}_{3} \mathrm{~N}$ & 0 \\
8 & 0.1 & 0 & 0 & $\mathrm{Et}_{3} \mathrm{~N}$ & $\mathrm{Et}_{3} \mathrm{~N}$ & 0 \\
9 & 0.1 & 0.2 & 0.2 & $\mathrm{Toluene}_{3}$ & $\mathrm{KOH}^{\mathrm{c}}$ & 0 \\
10 & 0.1 & 0.2 & 0.2 & DMF & $\mathrm{KOH}^{\mathrm{c}}$ & 0 \\
11 & 0.1 & 0.2 & 0.2 & Toluene & $\mathrm{K}_{2} \mathrm{CO}_{3}{ }^{\mathrm{c}}$ & 0 \\
12 & 0.1 & 0.2 & 0.2 & DMF & $\mathrm{K}_{2} \mathrm{CO}_{3}{ }^{\mathrm{c}}$ & 0 \\
\hline
\end{tabular}

${ }^{\mathrm{a}}$ Reaction conditions: [1a]:[2a]:[Pd] $=1000: 1100: 1$, at $50{ }^{\circ} \mathrm{C}$ for $3 \mathrm{~h} .{ }^{\mathrm{b}}$ Isolated yields. ${ }^{\mathrm{c}} 3$ equiv based on 1a was used as a base. 
The use of toluene, DMF, DMSO, and NMP as solvents resulting in lower rates of conversion (Entries 2-5). In the absence of triphenylphosphine, this Sonogashira coupling reaction still proceeded, but with less satisfactory yields (Entry 6). However, the co-catalyst $\mathrm{CuI}$ appeared to be necessary for the coupling reaction (Entries 7-8). Regarding the use of a base, inorganic bases such as $\mathrm{KOH}$ and $\mathrm{K}_{2} \mathrm{CO}_{3}$ were also examined, but under these conditions the desired product was not obtained due to the poor solubility of these salts in organic solvents (Entries 9-12) [55].

\subsection{Sonogashira reaction of aryl halides with phenylacetylene}

Following optimization of the reaction conditions, the reactions of $2 \mathbf{a}$ with various aryl halides were screened in the subsequent investigation (Table 2).

Table 2. Sonogashira reaction of aryl halides (1) with phenylacetylene (2a) catalyzed by nanosized MCM-41-Pd. ${ }^{\mathrm{a}}$

\begin{tabular}{|c|c|c|c|c|c|c|c|c|}
\hline Entry & Aryl halide & & Pd (mol\%) & Solvent/Base & $\mathrm{T}\left({ }^{\circ} \mathrm{C}\right)$ & t (h) & Yield $(\%)^{b}$ & TON \\
\hline 1 & $\mathrm{C}_{6} \mathrm{H}_{5} \mathrm{I}$ & $1 \mathbf{a}$ & 0.1 & $\mathrm{Et}_{3} \mathrm{~N} / \mathrm{Et}_{3} \mathrm{~N}$ & 50 & 3 & 3a, 97 & 970 \\
\hline 2 & $\mathrm{C}_{6} \mathrm{H}_{5} \mathrm{I}$ & 1a & 0.01 & $\mathrm{Et}_{3} \mathrm{~N} / \mathrm{Et}_{3} \mathrm{~N}$ & 50 & 12 & 3a, 98 & 9800 \\
\hline 3 & $4-\mathrm{IC}_{6} \mathrm{H}_{4} \mathrm{CN}$ & 1b & 0.1 & $\mathrm{Et}_{3} \mathrm{~N} / \mathrm{Et}_{3} \mathrm{~N}$ & 50 & 3 & $3 \mathbf{b}, 96$ & 960 \\
\hline 4 & $4-\mathrm{IC}_{6} \mathrm{H}_{4} \mathrm{CN}$ & 1b & 0.01 & $\mathrm{Et}_{3} \mathrm{~N} / \mathrm{Et}_{3} \mathrm{~N}$ & 50 & 9 & $3 \mathbf{b}, 96$ & 9600 \\
\hline 5 & $4-\mathrm{MeOC}_{6} \mathrm{H}_{4} \mathrm{I}$ & 1c & 0.1 & $\mathrm{Et}_{3} \mathrm{~N} / \mathrm{Et}_{3} \mathrm{~N}$ & 50 & 24 & 3c, 87 & 870 \\
\hline 6 & $\mathrm{C}_{6} \mathrm{H}_{5} \mathrm{Br}$ & 1d & 0.1 & $\mathrm{NMP} / \mathrm{Bu}_{3} \mathrm{~N}^{\mathrm{c}}$ & 140 & 24 & $3 a, 30$ & 300 \\
\hline 7 & $\mathrm{C}_{6} \mathrm{H}_{5} \mathrm{Br}$ & 1d & 0.1 & Toluene $/ \mathrm{Bu}_{3} \mathrm{~N}^{\mathrm{c}}$ & 100 & 24 & $3 a, 56$ & 560 \\
\hline 8 & $4-\mathrm{BrC}_{6} \mathrm{H}_{4} \mathrm{CN}$ & 1e & 0.1 & $\mathrm{Et}_{3} \mathrm{~N} / \mathrm{Et}_{3} \mathrm{~N}$ & 90 & 3 & $3 \mathbf{b}, 93$ & 930 \\
\hline 9 & 4- $\mathrm{MeCOC}_{6} \mathrm{H}_{4} \mathrm{Br}$ & 1f & 0.1 & $\mathrm{NMP} / \mathrm{Et}_{3} \mathrm{~N}^{\mathrm{c}}$ & 90 & 6 & 3d, 98 & 980 \\
\hline 10 & $4-\mathrm{NO}_{2} \mathrm{C}_{6} \mathrm{H}_{4} \mathrm{Br}$ & $1 \mathrm{~g}$ & 0.01 & $\mathrm{NMP} / \mathrm{Et}_{3} \mathrm{~N}^{\mathrm{c}}$ & 90 & 6 & 3e, 99 & 9900 \\
\hline 11 & $4-\mathrm{ClC}_{6} \mathrm{H}_{4} \mathrm{Br}$ & 1h & 0.1 & $\mathrm{NMP} / \mathrm{Et}_{3} \mathrm{~N}^{\mathrm{c}}$ & 90 & 24 & $3 f, 46$ & 460 \\
\hline 12 & $4-\mathrm{MeOC}_{6} \mathrm{H}_{4} \mathrm{Br}$ & $\mathbf{1 i}$ & 0.1 & $\mathrm{NMP} / \mathrm{Et}_{3} \mathrm{~N}^{\mathrm{c}}$ & 90 & 72 & $3 c, 40$ & 400 \\
\hline 13 & 2-Bromothiophene & $\mathbf{1 j}$ & 0.1 & $\mathrm{NMP} / \mathrm{Et}_{3} \mathrm{~N}^{\mathrm{c}}$ & 90 & 48 & $3 g, 71$ & 710 \\
\hline 14 & 3-Bromothiophene & $1 \mathbf{k}$ & 0.1 & $\mathrm{NMP} / \mathrm{Et}_{3} \mathrm{~N}^{\mathrm{c}}$ & 90 & 96 & $3 \mathbf{h}, 36$ & 360 \\
\hline 15 & 2-Bromopyridine & 11 & 0.1 & $\mathrm{NMP} / \mathrm{Et}_{3} \mathrm{~N}^{\mathrm{c}}$ & 90 & 3 & 3i, 99 & 990 \\
\hline 16 & 3-Bromopyridine & $1 \mathbf{m}$ & 0.1 & $\mathrm{NMP} / \mathrm{Et}_{3} \mathrm{~N}^{\mathrm{c}}$ & 90 & 24 & $3 \mathbf{j}, 98$ & 980 \\
\hline
\end{tabular}

${ }^{a}$ Reaction conditions: $[\mathbf{1}]:[2 a]=1: 1.1,[\mathrm{Pd}]:[\mathrm{CuI}]:\left[\mathrm{PPh}_{3}\right]=1: 2: 2 .{ }^{\mathrm{b}}$ Isolated yields. ${ }^{\mathrm{c}} 3$ equiv based on $\mathbf{1}$ was used as a base.

Reactions of 2a with aryl iodides proceeded well with the use of $0.1 \mathrm{~mol} \%$ NS-MCM-41-Pd at $50{ }^{\circ} \mathrm{C}$ (Table 2, Entries 1, 3, and 5), and it should be noted that the same good yields were also obtained in these reactions when using a lower amount of catalyst $(0.01 \mathrm{~mol} \%)$ (Entries 2 and 4). However, the Sonogashira reaction of bromobenzene (1d) and 2a under the same conditions did not afford any product, but replacing the $\mathrm{Et}_{3} \mathrm{~N}$ solvent by NMP, a typical solvent for such coupling reactions, resulted in the formation of $\mathbf{3 a}$ in a $30 \%$ yield at an elevated temperature (Entry 6), and a yield of up to $56 \%$ could be achieved by performing the reaction in toluene at $100{ }^{\circ} \mathrm{C}$ for $24 \mathrm{~h}$ (Entry 7). Using activated aryl bromides such as 4-bromobenzonitrile (1e), 4-bromoacetophenone (1f), and 4-bromonitrobenzene (19), better yields of the coupling reactions were observed (Entries 8-10). In the case of the coupling of $\mathbf{1 h}$ with $\mathbf{2 a}$, the $\mathrm{C}-\mathrm{Cl}$ bond was inert under the reaction conditions, while the product coupled through the $\mathrm{C}-\mathrm{Br}$ bond was obtained in a $46 \%$ yield (Entry 11 ). Next, we studied the coupling of halothiophenes and halopyridines with $\mathbf{2 a}$, and it appeared that 2-bromothiophene (1j) and 
2-bromo-pyridine (11) resulted in better yields than the corresponding bromides at the 3-position (Entries 13-16).

\subsection{Sonogashira reaction of aryl halides with alkynols}

Under similar conditions, NS-MCM-41-Pd-catalyzed Sonogashira coupling of a wide variety of aryl halides with 2-methyl-3-butyn-2-ol (4a) was also achieved (Table 3), and a reaction temperature of $90{ }^{\circ} \mathrm{C}$ was found to be optimal. Aryl iodides reacted with 4a to give the corresponding coupling products in good to excellent yields (Entries 1-3), whereas the use of deactivated bromides as substrates resulted in lower yields (Entry 4). Reactions of activated bromides delivered better conversion rates (Entries 5-9): for example, the catalyst had a turnover number (TON) of 9,600 for the coupling of 1e with 4a (Entry 7), and for the heteroaryl halides (Entries 11-15), the catalyst exhibited great activity, with the exception of the reactions of $\mathbf{1 k}$ (Entries 12).

Table 3. Sonogashira reaction of aryl halides 1 with alkynols 4 catalyzed by nanosized MCM-41-Pd. ${ }^{\text {a }}$

\begin{tabular}{|c|c|c|c|c|c|c|c|c|}
\hline Entry & Aryl halide & & Alkynyl alcohol & & $\operatorname{Pd}(\mathrm{mol} \%)$ & $t(\mathrm{~h})$ & Yield $(\%)^{\mathrm{b}}$ & TON \\
\hline 1 & $\mathrm{C}_{6} \mathrm{H}_{5} \mathrm{I}$ & $1 \mathbf{a}$ & $\mathrm{HC} \equiv \mathrm{CC}\left(\mathrm{CH}_{3}\right)_{2} \mathrm{OH}$ & $4 a$ & 0.1 & 3 & $5 a, 94$ & 940 \\
\hline 2 & $4-\mathrm{IC}_{6} \mathrm{H}_{4} \mathrm{CN}$ & $1 b$ & $\mathrm{HC} \equiv \mathrm{CC}\left(\mathrm{CH}_{3}\right)_{2} \mathrm{OH}$ & $4 a$ & 0.1 & 3 & $5 \mathbf{b}, 98$ & 980 \\
\hline 3 & 4- $\mathrm{MeOC}_{6} \mathrm{H}_{4} \mathrm{I}$ & 1c & $\mathrm{HC} \equiv \mathrm{CC}\left(\mathrm{CH}_{3}\right)_{2} \mathrm{OH}$ & $4 a$ & 0.1 & 72 & $5 c, 61$ & 610 \\
\hline 4 & $\mathrm{C}_{6} \mathrm{H}_{5} \mathrm{Br}$ & 1d & $\mathrm{HC} \equiv \mathrm{CC}\left(\mathrm{CH}_{3}\right)_{2} \mathrm{OH}$ & $4 a$ & 0.1 & 96 & $5 a, 21$ & 210 \\
\hline 5 & 4- $\mathrm{BrC}_{6} \mathrm{H}_{4} \mathrm{CN}$ & 1e & $\mathrm{HC} \equiv \mathrm{CC}\left(\mathrm{CH}_{3}\right)_{2} \mathrm{OH}$ & $4 a$ & 0.1 & 3 & $5 \mathbf{b}, 98$ & 980 \\
\hline 6 & $4-\mathrm{BrC}_{6} \mathrm{H}_{4} \mathrm{CN}$ & 1e & $\mathrm{HC} \equiv \mathrm{CC}\left(\mathrm{CH}_{3}\right)_{2} \mathrm{OH}$ & $4 a$ & 0.01 & 12 & $5 \mathbf{b}, 96$ & 9600 \\
\hline 7 & 4- $\mathrm{MeCOC}_{6} \mathrm{H}_{4} \mathrm{Br}$ & 1f & $\mathrm{HC} \equiv \mathrm{CC}\left(\mathrm{CH}_{3}\right)_{2} \mathrm{OH}$ & $4 a$ & 0.1 & 3 & $5 d, 98$ & 980 \\
\hline 8 & $4-\mathrm{NO}_{2} \mathrm{C}_{6} \mathrm{H}_{4} \mathrm{Br}$ & $1 \mathrm{~g}$ & $\mathrm{HC} \equiv \mathrm{CC}\left(\mathrm{CH}_{3}\right)_{2} \mathrm{OH}$ & $4 a$ & 0.1 & 3 & $5 e, 97$ & 970 \\
\hline 9 & $4-\mathrm{ClC}_{6} \mathrm{H}_{4} \mathrm{Br}$ & 1h & $\mathrm{HC} \equiv \mathrm{CC}\left(\mathrm{CH}_{3}\right)_{2} \mathrm{OH}$ & $4 a$ & 0.1 & 24 & 5f, 69 & 690 \\
\hline 10 & 4- $\mathrm{MeOC}_{6} \mathrm{H}_{4} \mathrm{Br}$ & $1 \mathbf{i}$ & $\mathrm{HC} \equiv \mathrm{CC}\left(\mathrm{CH}_{3}\right)_{2} \mathrm{OH}$ & $4 a$ & 0.1 & 96 & $5 c, 20$ & 200 \\
\hline 11 & 2-Bromothiophene & $\mathbf{1 j}$ & $\mathrm{HC} \equiv \mathrm{CC}\left(\mathrm{CH}_{3}\right)_{2} \mathrm{OH}$ & $4 a$ & 0.1 & 48 & $5 g, 99$ & 990 \\
\hline 12 & 3-Bromothiophene & $1 \mathbf{k}$ & $\mathrm{HC} \equiv \mathrm{CC}\left(\mathrm{CH}_{3}\right)_{2} \mathrm{OH}$ & $4 a$ & 0.1 & 96 & $5 \mathbf{h}, 59$ & 590 \\
\hline 13 & 2-Bromopyridine & 11 & $\mathrm{HC} \equiv \mathrm{CC}\left(\mathrm{CH}_{3}\right)_{2} \mathrm{OH}$ & $4 a$ & 0.1 & 3 & $5 \mathbf{i}, 99$ & 990 \\
\hline 14 & 3-Bromopyridine & $1 \mathrm{~m}$ & $\mathrm{HC} \equiv \mathrm{CC}\left(\mathrm{CH}_{3}\right)_{2} \mathrm{OH}$ & $4 a$ & 0.1 & 6 & $5 \mathbf{j}, 98$ & 980 \\
\hline 15 & 3-Bromopyridine & $1 \mathrm{~m}$ & $\mathrm{HC} \equiv \mathrm{CC}\left(\mathrm{CH}_{3}\right)_{2} \mathrm{OH}$ & $4 a$ & 0.01 & 24 & $5 \mathbf{j}, 34$ & 3400 \\
\hline 16 & $\mathrm{C}_{6} \mathrm{H}_{5} \mathrm{I}$ & $1 \mathbf{a}$ & $\mathrm{HC} \equiv \mathrm{CCH}_{2} \mathrm{OH}$ & $4 b$ & 0.1 & 12 & $5 \mathbf{k}, 85$ & 850 \\
\hline 17 & $\mathrm{C}_{6} \mathrm{H}_{5} \mathrm{I}$ & 1a & $\mathrm{HC} \equiv \mathrm{CCH}_{2} \mathrm{OH}$ & $4 b$ & 0.01 & 24 & $5 \mathbf{k}, 84$ & 8400 \\
\hline 18 & 4- $\mathrm{IC}_{6} \mathrm{H}_{4} \mathrm{CN}$ & $1 \mathrm{~b}$ & $\mathrm{HC} \equiv \mathrm{CCH}_{2} \mathrm{OH}$ & $4 b$ & 0.1 & 3 & $5 \mathbf{l}, 83$ & 830 \\
\hline 19 & $\mathrm{C}_{6} \mathrm{H}_{5} \mathrm{Br}$ & 1d & $\mathrm{HC} \equiv \mathrm{CCH}_{2} \mathrm{OH}$ & $4 b$ & 0.1 & 96 & $5 \mathbf{k}, 10$ & 100 \\
\hline 20 & $4-\mathrm{BrC}_{6} \mathrm{H}_{4} \mathrm{CN}$ & $1 e$ & $\mathrm{HC} \equiv \mathrm{CCH}_{2} \mathrm{OH}$ & $4 b$ & 0.1 & 24 & $5 \mathbf{l}, 71$ & 710 \\
\hline 21 & 4- $\mathrm{BrC}_{6} \mathrm{H}_{4} \mathrm{COMe}$ & 1f & $\mathrm{HC} \equiv \mathrm{CCH}_{2} \mathrm{OH}$ & $4 b$ & 0.1 & 3 & $5 \mathbf{m}, 98$ & 980 \\
\hline 22 & 4- $\mathrm{BrC}_{6} \mathrm{H}_{4} \mathrm{COMe}$ & 1f & $\mathrm{HC} \equiv \mathrm{CCH}_{2} \mathrm{OH}$ & $4 b$ & 0.01 & 48 & $5 \mathbf{m}, 98$ & 9800 \\
\hline 23 & 4- $\mathrm{BrC}_{6} \mathrm{H}_{4} \mathrm{NO}_{2}$ & $1 \mathrm{~g}$ & $\mathrm{HC} \equiv \mathrm{CCH}_{2} \mathrm{OH}$ & $4 b$ & 0.1 & 24 & $5 n, 99$ & 990 \\
\hline 24 & $4-\mathrm{BrC}_{6} \mathrm{H}_{4} \mathrm{Cl}$ & 1h & $\mathrm{HC} \equiv \mathrm{CCH}_{2} \mathrm{OH}$ & $4 b$ & 0.1 & 72 & 50,15 & 150 \\
\hline 25 & 2-Bromothiophene & $\mathbf{1 j}$ & $\mathrm{HC} \equiv \mathrm{CCH}_{2} \mathrm{OH}$ & $4 b$ & 0.1 & 48 & $5 p, 18$ & 180 \\
\hline
\end{tabular}


Table 3. Cont.

\begin{tabular}{lllllllll}
\hline 26 & 3-Bromothiophene & $\mathbf{1 k}$ & $\mathrm{HC} \equiv \mathrm{CCH}_{2} \mathrm{OH}$ & $\mathbf{4 b}$ & 0.1 & 96 & $\mathbf{5 q}, 8$ & 80 \\
27 & 2-Bromopyridine & $\mathbf{1 l}$ & $\mathrm{HC} \equiv \mathrm{CCH}_{2} \mathrm{OH}$ & $\mathbf{4 b}$ & 0.1 & 3 & $\mathbf{5 r}, 81$ & 810 \\
28 & 3-Bromopyridine & $\mathbf{1 m}$ & $\mathrm{HC} \equiv \mathrm{CCH}_{2} \mathrm{OH}$ & $\mathbf{4 b}$ & 0.1 & 48 & $\mathbf{5 s , 6 5}$ & 650 \\
29 & $\mathrm{C}_{6} \mathrm{H}_{5} \mathrm{I}$ & $\mathbf{1 a}$ & $\mathrm{HC} \equiv \mathrm{CCH}_{2} \mathrm{CH}_{2} \mathrm{OH}$ & $\mathbf{4 c}$ & 0.1 & 6 & $\mathbf{5 t}, 78$ & 780 \\
30 & 4- $\mathrm{MeOC}_{6} \mathrm{H}_{4} \mathrm{I}$ & $\mathbf{1 c}$ & $\mathrm{HC} \equiv \mathrm{CCH}_{2} \mathrm{CH}_{2} \mathrm{OH}$ & $\mathbf{4 c}$ & 0.1 & 12 & $\mathbf{5 u}, 45$ & 450 \\
31 & 4- $\mathrm{BrC}_{6} \mathrm{H}_{4} \mathrm{CN}$ & $\mathbf{1 e}$ & $\mathrm{HC} \equiv \mathrm{CCH}_{2} \mathrm{CH}_{2} \mathrm{OH}$ & $\mathbf{4 c}$ & 0.1 & 12 & $\mathbf{5 v}, 99$ & 990 \\
32 & 4- $\mathrm{BrC}_{6} \mathrm{H}_{4} \mathrm{COMe}$ & $\mathbf{1 f}$ & $\mathrm{HC} \equiv \mathrm{CCH}_{2} \mathrm{CH}_{2} \mathrm{OH}$ & $\mathbf{4 c}$ & 0.1 & 12 & $\mathbf{5 w}, 89$ & 890 \\
33 & 2-Bromothiophene & $\mathbf{1 j}$ & $\mathrm{HC} \equiv \mathrm{CCH}_{2} \mathrm{CH}_{2} \mathrm{OH}$ & $\mathbf{4 c}$ & 0.1 & 12 & $\mathbf{5 x}, 32$ & 320 \\
34 & 2-Bromopyridine & $\mathbf{1 l}$ & $\mathrm{HC} \equiv \mathrm{CCH}_{2} \mathrm{CH}_{2} \mathrm{OH}$ & $\mathbf{4 c}$ & 0.1 & 12 & $\mathbf{5 y}, 64$ & 640 \\
\hline
\end{tabular}

${ }^{\mathrm{a}}$ Reaction conditions: $[\mathbf{1}]:[\mathbf{4 a}$ or $\mathbf{4 c}]=1: 1.1 ;[\mathbf{1}]:[\mathbf{4 b}]=1: 1.5 ;[\mathrm{Pd}]:[\mathrm{CuI}]:\left[\mathrm{PPh}_{3}\right]=1: 2: 2 ; \mathrm{Et}_{3} \mathrm{~N}$ was used as the solvent and base at $90{ }^{\circ} \mathrm{C} .{ }^{\mathrm{b}}$ Isolated yields.

We also studied the reactivity of propargyl alcohol (4b) with aryl and heteroaryl halides in the presence of $0.1-0.01 \mathrm{~mol} \%$ of catalyst (Entries 16-28). Generally, the reaction rates for the coupling of aryl and heteroaryl halides with $\mathbf{4 b}$ were slower than those for coupling with $\mathbf{4 a}$, and reaction of aryl iodides with $\mathbf{4 b}$ at $90{ }^{\circ} \mathrm{C}$ gave the desired products in high yields (Entries 16-18). The use of 1d afforded only $10 \%$ of product under the reaction conditions described (Entry 19), while for electronpoor aryl bromides, excellent yields were obtained (Entries 20-23). In the case of 1f, using a $0.01 \mathrm{~mol} \%$ catalyst loading, a TON of 9,800 was achieved, which is comparable with the reported efficiency of homogeneous catalysts [17b,f,g] (Entry 22). On the other hand, coupling of $\mathbf{1 h}$ with $\mathbf{4 b}$ gave a yield of only $15 \%$ (Entry 24). As for heteroaryl halides, the use of bromothiophenes $\mathbf{1} \mathbf{j}$ and $\mathbf{1 k}$ did not provide the products in good yields (Entries 25 and 26), while with halopyridines, the coupling products were obtained in good to excellent yields (Entries 27 and 28). The coupling of 3-butyn-1-ol (4c) with aryl and heteroaryl halides at a catalyst loading of $0.1 \mathrm{~mol} \%$ was also screened, and the corresponding products were obtained in moderate to high yields (Entries 29-34).

\subsection{Recycling and leaching studies of NS-MCM-41-Pd in the Sonogashira reaction}

One of the purposes of designing this catalyst was to enable catalyst recycling for further use in subsequent reactions. In context, aryl iodides, activated aryl bromides, and several terminal alkynes were examined under optimized reaction conditions, and after completion of the initial cycle, the NSMCM-41-Pd catalyst was extracted by centrifugation from the reaction mixture, washed successively with THF, $\mathrm{H}_{2} \mathrm{O}$, and THF, and used for the next run with no regeneration treatment. The results using the recycled catalyst are shown in Table 4 . In the case of aryl iodides, we found that the activity of the catalyst was completely retained after two recycled runs, giving an overall TON of between 2,450 and 2,960 (Table 4, Entries 1, 3, and 5). For the activated aryl bromides, the NS-MCM-41-Pd catalyst also exhibited high TONs in the recycled runs (Entries 2 and 4), but a gradual decrease in catalytic activity was observed in the reaction of $\mathbf{1 f}$ with $\mathbf{4 b}$ (Entry 6 ). 
Table 4. Sonogashira coupling reaction catalyzed by recycled nanosized MCM-41-Pd. ${ }^{\mathrm{a}}$

\begin{tabular}{|c|c|c|c|c|c|c|c|c|}
\hline \multirow{2}{*}{ Entry } & \multirow{2}{*}{ Aryl halide } & \multirow{2}{*}{ Alkyne } & \multirow{2}{*}{ Solvent/Base } & \multirow{2}{*}{$\mathbf{T}\left({ }^{\circ} \mathbf{C}\right)$} & \multirow{2}{*}{ t (h) } & \multicolumn{3}{|c|}{ Yield, \% ${ }^{b}$ (TON) } \\
\hline & & & & & & Initial cycle & 1st recycle & 2nd recycle \\
\hline 1 & $1 a$ & $2 a$ & $\mathrm{Et}_{3} \mathrm{~N} / \mathrm{Et}_{3} \mathrm{~N}$ & 50 & 3 & $99(990)$ & $99(990)$ & $98(980)$ \\
\hline 2 & 1f & $2 a$ & $\mathrm{NMP} / \mathrm{Et}_{3} \mathrm{~N}^{\mathrm{c}}$ & 90 & 6 & $98(980)$ & $93(930)$ & $91(910)$ \\
\hline 3 & 1a & $4 a$ & $\mathrm{Et}_{3} \mathrm{~N} / \mathrm{Et}_{3} \mathrm{~N}$ & 90 & 3 & $94(940)$ & $90(900)$ & $88(880)$ \\
\hline 4 & 1f & $4 \mathbf{a}$ & $\mathrm{Et}_{3} \mathrm{~N} / \mathrm{Et}_{3} \mathrm{~N}$ & 90 & 3 & $98(980)$ & $99(990)$ & $95(950)$ \\
\hline $5^{d}$ & $1 b$ & $4 \mathbf{b}$ & $\mathrm{Et}_{3} \mathrm{~N} / \mathrm{Et}_{3} \mathrm{~N}$ & 90 & 3 & $83(830)$ & $82(820)$ & $80(800)$ \\
\hline $6^{d}$ & 1f & $4 \mathbf{b}$ & $\mathrm{Et}_{3} \mathrm{~N} / \mathrm{Et}_{3} \mathrm{~N}$ & 90 & 3 & $98(980)$ & $88(880)$ & $76(760)$ \\
\hline
\end{tabular}

Several studies have successfully determined the amount of metal leaching using a hot-filtration technique, and this method was therefore used in this study to examine the activity of the catalyst with regards to metal leaching [45-48,56-58]. A reaction mixture of 1a with 2a in the above-described catalytic system was stirred at $50{ }^{\circ} \mathrm{C}$ for $30 \mathrm{~min}$, resulting in a $\mathrm{GC}$ yield of $32 \%$. The hot reaction mixture was then filtered through a dried Celite pad under nitrogen to remove the NS-MCM-41-Pd catalyst and any insoluble species, and the clear filtrate was introduced to another Schlenk tube at $50{ }^{\circ} \mathrm{C}$. Further detection by GC demonstrated improvement of the yield to only $37 \%$ after $3 \mathrm{~h}$, even in the presence of additional $\mathrm{CuI}$ and $\mathrm{PPh}_{3}$ (Figure 2). This result shows that no active species were dissolved in the solution to catalyze the coupling reaction. We further determined the Pd-content in the filtrate by ICP-MASS, and only $0.5 \mathrm{ppm}$ of palladium was found in the solution, which indicated that the catalytic activity may mainly result from the grafted palladium complex. However, another pathway of the Sonogashira reaction is catalysis by a dissolved Pd-species that occurs inside the channels of NS-MCM-41, and this pathway cannot be excluded.

Figure 2. Plot of yield versus time with hot-filtration for $0.5 \mathrm{~h}$ of reaction at $50{ }^{\circ} \mathrm{C}(\boldsymbol{m})$ and

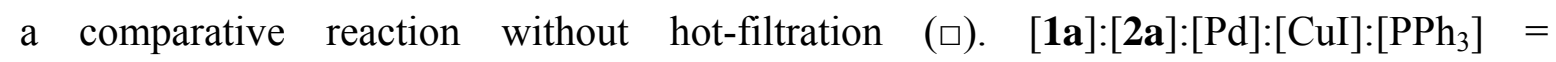
$1000 / 1100 / 1 / 2 / 2$.

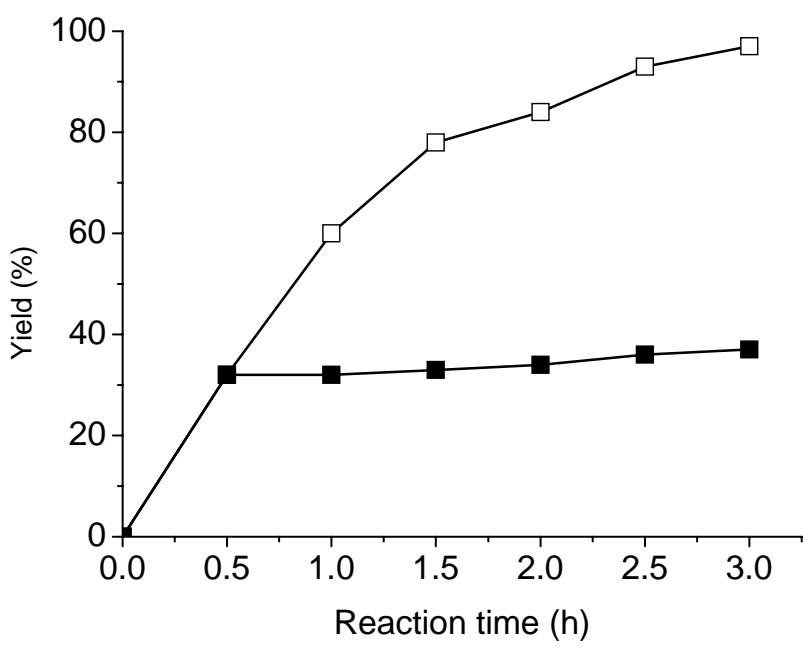




\section{Experimental}

\subsection{General}

All reactions involving air- and moisture- sensitive conditions were carried out under a dry nitrogen atmosphere. $\mathrm{N}$-Methylpyrrolidinone (NMP) was distilled under reduced pressure before use; $\mathrm{Et}_{3} \mathrm{~N}$ and $\mathrm{Bu}_{3} \mathrm{~N}$ were distilled from $\mathrm{KOH}$; and toluene was distilled from sodium benzophenone ketyl. Aryl halides and terminal alkynes were purchased from ARCOS Co. Ltd and were used without further purification. 4,4'-Bis(bromomethyl)-2,2'-bipyridine [59,60], nanosized MCM-41 [61], and NS-MCM41-Pd [55,56] were prepared according to the previously-published procedures. Melting points were recorded on melting point apparatus and were uncorrected. ${ }^{1} \mathrm{H}$ - and ${ }^{13} \mathrm{C}-\mathrm{NMR}$ spectra were recorded in $\mathrm{CDCl}_{3}$ or $\mathrm{C}_{6} \mathrm{D}_{6}$ solution at $25^{\circ} \mathrm{C}$ on a Varian $200 \mathrm{NMR}$ spectrometer. GC analysis was performed on an SRI $8610 \mathrm{C}$ instrument equipped with a fused silica capillary column.

\subsection{General procedure for the Sonogashira coupling}

Under a nitrogen atmosphere, a mixture of NS-MCM-41-Pd (50 mg, $\left.7.5 \times 10^{-3} \mathrm{mmol}-\mathrm{Pd}\right)$, CuI (2.9 mg, $\left.1.5 \times 10^{-2} \mathrm{mmol}\right)$, and $\mathrm{PPh}_{3}\left(3.9 \mathrm{mg}, 1.5 \times 10^{-2} \mathrm{mmol}\right)$ in $\mathrm{Et}_{3} \mathrm{~N}(15 \mathrm{~mL})$ in a $50 \mathrm{~mL}$ Schlenk tube was charged with aryl halide $(7.5 \mathrm{mmol})$ and terminal alkyne $(8.3 \mathrm{mmol}$; in the case of $\mathbf{4 b}$, $11.3 \mathrm{mmol}$ was used), and the reaction mixture stirred at $50{ }^{\circ} \mathrm{C}$ or $90{ }^{\circ} \mathrm{C}$. After cooling to room temperature, the resulting solution was passed through a short silica gel column with ethyl acetate as the eluent to remove ammonium salt. After evaporation of the solvent, column chromatography on silica gel afforded the desired product.

Diphenylacetylene (3a). White solid. m.p. 60-61 ${ }^{\circ} \mathrm{C}$ (lit.[47] 60-61 ${ }^{\circ} \mathrm{C}$ ). ${ }^{1} \mathrm{H}-\mathrm{NMR}: \delta$ 7.32-7.34 (m, 6H), 7.51-7.56 (m, 4H); ${ }^{13} \mathrm{C}-\mathrm{NMR}: \delta 89.2(2 \mathrm{C}), 122.9(2 \mathrm{C}), 127.8(4 \mathrm{C}), 127.9(2 \mathrm{C}), 131.2(4 \mathrm{C})$.

4-(Phenylethynyl)benzonitrile (3b). Pale yellow solid. m.p. $108-110{ }^{\circ} \mathrm{C}$ (lit.[62] $\left.106-108{ }^{\circ} \mathrm{C}\right) .{ }^{1} \mathrm{H}-$ NMR: $\delta 7.35-7.38(\mathrm{~m}, 3 \mathrm{H}), 7.51-7.55(\mathrm{~m}, 2 \mathrm{H}), 7.60-7.61(\mathrm{~m}, 4 \mathrm{H}) ;{ }^{13} \mathrm{C}-\mathrm{NMR}: \delta 87.6,93.6,111.1$, $118.2,121.8,127.8,128.1$ (2C), 128.7, 131.3 (2C), $131.6(2 \mathrm{C}), 131.6(2 \mathrm{C})$.

4-(Phenylethynyl)anisole (3c). Brown solid. m.p. $60-61{ }^{\circ} \mathrm{C}$ (lit.[62] 60.6 $\left.{ }^{\circ} \mathrm{C}\right) .{ }^{1} \mathrm{H}-\mathrm{NMR}: \delta 2.88(\mathrm{~s}, 3 \mathrm{H})$, 6.28-6.33 (m, 2H), 6.69-6.70 (m, 3H), 7.15-7.20 (m, 4H); ${ }^{13} \mathrm{C}-\mathrm{NMR}: \delta$ 55.4, 87.9 (2C), $113.7(2 \mathrm{C})$, $115.0,123.2,127.5,127.9(2 \mathrm{C}), 131.0(2 \mathrm{C}), 132.6(2 \mathrm{C}), 158.9$.

4-(Phenylethynyl)acetophenone (3d). Brown solid. m.p. 97-99 ${ }^{\circ} \mathrm{C}$ (lit.[47] 98-99 $\left.{ }^{\circ} \mathrm{C}\right) .{ }^{1} \mathrm{H}-\mathrm{NMR}: \delta$ $2.60(\mathrm{~s}, 3 \mathrm{H}), 7.34-7.37(\mathrm{~m}, 3 \mathrm{H}), 7.51-7.61(\mathrm{~m}, 2 \mathrm{H}), 7.78-7.83(\mathrm{~m}, 2 \mathrm{H}), 7.91-7.95(\mathrm{~m}, 2 \mathrm{H})$; ${ }^{13} \mathrm{C}-\mathrm{NMR}$ : $\delta 27.0,88.5,92.6,122.3,127.8,127.9$ (2C), $128.0(2 \mathrm{C}), 128.4,131.3$ (2C), $132.0(2 \mathrm{C}), 135.7,196.4$.

4-(Phenylethynyl)nitrobenzene (3e). Yellow solid. m.p. 116-117 ${ }^{\circ} \mathrm{C}$ (lit.[62] $\left.114-116{ }^{\circ} \mathrm{C}\right) .{ }^{1} \mathrm{H}-\mathrm{NMR}$ : $\delta$ 7.34-7.39 (m, 3H) 7.52-7.56 (m, 2H), 7.63-7.66 (m, 2H), 8.19-8.22 (m, 2H); ${ }^{13} \mathrm{C}-\mathrm{NMR}: \delta 87.4,94.6$, $121.7,123.3,128.1(2 \mathrm{C}), 128.9$ (2C), $129.8,131.4(2 \mathrm{C}), 131.8(2 \mathrm{C}), 146.4$. 
Phenyl-(4-chlorophenyl)acetylene (3f). White solid. m.p. 82-83 ${ }^{\circ} \mathrm{C}$ (lit.[47] 82-83 $\left.{ }^{\circ} \mathrm{C}\right) .{ }^{1} \mathrm{H}-\mathrm{NMR}: \delta$ 7.30-7.36 (m, 5H), 7.43-7.46 (m, 2H), 7.51-7.53 (m, 2H); ${ }^{13} \mathrm{C}-\mathrm{NMR}: \delta 88.1,90.2,121.4,122.5,128.0$ (2C), 128.1, 128.3 (2C), $131.2(2 \mathrm{C}), 132.4(2 \mathrm{C}), 133.8$.

2-(Theinylethynyl)benzene (3g). Pale yellow solid. m.p. 50-52 ${ }^{\circ} \mathrm{C}$ (lit.[63] 51-53 $\left.{ }^{\circ} \mathrm{C}\right) .{ }^{1} \mathrm{H}-\mathrm{NMR}: \delta$ 7.00-7.02 (m, 1H), 7.28-7.29 (m, 2H), 7.34-7.35 (m, 3H), 7.51-7.52 (m, 2H); ${ }^{13} \mathrm{C}-\mathrm{NMR}: \delta 82.5,92.9$, $126.7,126.8,127.9$ (2C), 128.0, 128.1, $131.0(2 \mathrm{C}), 131.5,132.0$.

3-(Theinylethynyl)benzene (3h). Brown solid. m.p. 50-52 ${ }^{\circ} \mathrm{C}$ (lit.[64] 52-54 $\left.{ }^{\circ} \mathrm{C}\right) .{ }^{1} \mathrm{H}-\mathrm{NMR}: \delta$ 7.20-7.22 (m, 1H), 7.29-7.32 (m, 1H), 7.33-7.37 (m, 3H), 7.52-7.54 (m, 3H); ${ }^{13} \mathrm{C}-\mathrm{NMR}: \delta 84.4,88.8$, $121.9,122.8,125.0,127.8,127.9(2 \mathrm{C}), 128.2,129.4,131.1(2 \mathrm{C})$.

2-(Phenylethynyl)pyridine (3i) [65]. Colorless liquid. ${ }^{1} \mathrm{H}-\mathrm{NMR}: \delta$ 7.15-7.18 (m, 1H), 7.29-7.32 (m, 3H), 7.45-7.47 (m, 1H), 7.54-7.62 (m, 3H), 8.55-8.57 (m, $1 \mathrm{H}) ;{ }^{13} \mathrm{C}-\mathrm{NMR}: \delta 88.4,88.9,121.7,122.3$, $126.6,127.9,128.4,131.5,135.6,142.8,149.3$.

3-(Phenylethynyl)pyridine (3j). Yellow solid. m.p. 50-51 ${ }^{\circ} \mathrm{C}$ (lit.[66] 50-51 $\left.{ }^{\circ} \mathrm{C}\right) .{ }^{1} \mathrm{H}-\mathrm{NMR}: \delta$ 7.23-7.26 (m, 1H), 7.33-7.36 (m, 3H), 7.52-7.55 (m, 2H), 7.76-7.79 (m, 1H), 8.51-8.53 (m, 1H), 8.75-8.76 (m, 1H); ${ }^{13} \mathrm{C}-\mathrm{NMR}: \delta 85.8,92.4,121.4,120.0,122.0,122.6,128.0(2 \mathrm{C}), 128.3,131.2(2 \mathrm{C})$, 137.8, 147.9, 151.6 .

2-Methyl-4-phenyl-3-butyn-2-ol (5a). Yellow solid. m.p. 53-54 ${ }^{\circ} \mathrm{C}$ (lit.[67] 53.5-54.5 $\left.{ }^{\circ} \mathrm{C}\right) .{ }^{1} \mathrm{H}-\mathrm{NMR}: \delta$ $1.61(\mathrm{~s}, 6 \mathrm{H}), 2.01(\mathrm{~s}, 1 \mathrm{H}), 7.27-7.28(\mathrm{~m}, 3 \mathrm{H}), 7.38-7.41(\mathrm{~m}, 2 \mathrm{H}) ;{ }^{13} \mathrm{C}-\mathrm{NMR}: \delta 31.8(2 \mathrm{C}), 65.7,82.1$, $104.1,122.3,127.8(2 \mathrm{C}), 127.9,131.2(2 \mathrm{C})$.

2-Methyl-4-(4'-cyano)phenyl-3-butyn-2-ol (5b) [68]. Yellow solid. m.p. 69-70 ${ }^{\circ} \mathrm{C}$ (lit.[69] 68.5-69.5 $\left.{ }^{\circ} \mathrm{C}\right) .{ }^{1} \mathrm{H}-\mathrm{NMR}: \delta 1.62(\mathrm{~s}, 6 \mathrm{H}), 2.12(\mathrm{~s}, 1 \mathrm{H}), 7.47(\mathrm{~d}, J=6.4 \mathrm{~Hz}, 2 \mathrm{H}), 7.57(\mathrm{~d}, J=6.4 \mathrm{~Hz}$, $2 \mathrm{H}) ;{ }^{13} \mathrm{C}-\mathrm{NMR}: \delta 31.6(2 \mathrm{C}), 65.7,80.6,98.0,111.3,118.1,127.3,131.5(2 \mathrm{C}), 131.7(2 \mathrm{C})$.

2-Methyl-4-(4'-methoxy)phenyl-3-butyn-2-ol (5c) [70]. Yellow oil. ${ }^{1} \mathrm{H}-\mathrm{NMR}: \delta 1.59$ (s, 6H), 2.24 (s, $1 \mathrm{H}), 3.76(\mathrm{~s}, 3 \mathrm{H}), 6.79(\mathrm{~d}, J=8.0 \mathrm{~Hz}, 2 \mathrm{H}), 7.31(\mathrm{~d}, J=8.0 \mathrm{~Hz}, 2 \mathrm{H}) ;{ }^{13} \mathrm{C}-\mathrm{NMR}: \delta 31.8(2 \mathrm{C}), 55.3,65.6$, $81.8,92.3,113.5(2 \mathrm{C}), 114.5,132.5(2 \mathrm{C}), 158.7$.

2-Methyl-4-(4'-acetyl)phenyl-3-butyn-2-ol (5d) [70]. Yellow oil. ${ }^{1} \mathrm{H}-\mathrm{NMR}: \delta 1.59$ (s, 6H), 2.53 (s, $3 \mathrm{H}), 2.83(\mathrm{~s}, 1 \mathrm{H}), 7.40(\mathrm{~d}, J=6.8 \mathrm{~Hz}, 2 \mathrm{H}), 7.81(\mathrm{~d}, J=6.8 \mathrm{~Hz}, 2 \mathrm{H}) ;{ }^{13} \mathrm{C}-\mathrm{NMR}: \delta 26.9,31.5(2 \mathrm{C}), 65.4$, 81.0, 97.1, 127.3, 127.7 (2C), $131.2(2 \mathrm{C}), 135.5,196.6$.

2-Methyl-4-(4'-nitro)phenyl-3-butyn-2-ol (5e). Brown solid. m.p. $100-102{ }^{\circ} \mathrm{C}$ (lit.[71] $102{ }^{\circ} \mathrm{C}$ ). ${ }^{1} \mathrm{H}-$ NMR: $\delta 1.62(\mathrm{~s}, 6 \mathrm{H}), 2.09$ (s, 1H), 7.49 (d, $J=7.6 \mathrm{~Hz}, 2 \mathrm{H}), 8.14(\mathrm{~d}, J=7.6 \mathrm{~Hz}, 2 \mathrm{H}) ;{ }^{13} \mathrm{C}-\mathrm{NMR}: \delta 31.5$ (2C), 65.7, 80.4, 99.8, 123.1 (2C), 129.3, 131.9 (2C), 146.5. 
2-Methyl-4-(4'-chloro)phenyl-3-butyn-2-ol (5f). White solid. m.p. 55-56 ${ }^{\circ} \mathrm{C}$ (lit.[72] $\left.55-57{ }^{\circ} \mathrm{C}\right) .{ }^{1} \mathrm{H}-$ NMR: $\delta 1.60(\mathrm{~s}, 6 \mathrm{H}), 2.11(\mathrm{~s}, 1 \mathrm{H}), 7.25(\mathrm{~d}, J=8.4 \mathrm{~Hz}, 2 \mathrm{H}), 7.31(\mathrm{~d}, J=8.4 \mathrm{~Hz}, 2 \mathrm{H}) ;{ }^{13} \mathrm{C}-\mathrm{NMR}: \delta 31.7$ (2C), 65.7, 81.0, 94.5, 120.8, $128.2(2 \mathrm{C}), 132.4$ (2C), 133.8.

2-Methyl-4-(2-thienyl)-3-butyn-2-ol (5g). Off-white solid. m.p. 56-57 ${ }^{\circ} \mathrm{C}$ (lit.[73] $\left.54{ }^{\circ} \mathrm{C}\right) .{ }^{1} \mathrm{H}-\mathrm{NMR}: \delta$ $1.58(\mathrm{~s}, 6 \mathrm{H}), 2.51(\mathrm{~s}, 1 \mathrm{H}), 6.91-6.93(\mathrm{~m}, 1 \mathrm{H}), 7.14-7.15(\mathrm{~m}, 1 \mathrm{H}), 7.19-7.21(\mathrm{~m}, 1 \mathrm{H}) ;{ }^{13} \mathrm{C}-\mathrm{NMR}: \delta 31.6$ (2C), 65.7, 75.4, 97.3, 122.2, 126.5, 126.6, 131.5 .

2-Methyl-4-(3-thienyl)-3-butyn-2-ol (5h). Brown solid. m.p. 54-56 ${ }^{\circ} \mathrm{C}$ (lit.[74] $\left.56{ }^{\circ} \mathrm{C}\right) .{ }^{1} \mathrm{H}-\mathrm{NMR}: \delta$ $1.61(\mathrm{~s}, 6 \mathrm{H}), 2.12(\mathrm{~s}, 1 \mathrm{H}), 7.08-7.09(\mathrm{~m}, 1 \mathrm{H}), 7.24-7.26(\mathrm{~m}, 1 \mathrm{H}), 7.41-7.42(\mathrm{~m}, 1 \mathrm{H}) ;{ }^{13} \mathrm{C}-\mathrm{NMR}: \delta 31.8$ (2C), 65.7, 77.3, 93.2, 124.9, 128.2, 129.4.

2-Methyl-4-(2-pyridyl)-3-butyn-2-ol (5i). Off-white solid. m.p. $60-61{ }^{\circ} \mathrm{C}$ (lit.[25] $\left.61{ }^{\circ} \mathrm{C}\right) .{ }^{1} \mathrm{H}-\mathrm{NMR}: \delta$ $1.59(\mathrm{~s}, 6 \mathrm{H}), 2.95(\mathrm{~s}, 1 \mathrm{H}), 7.11-7.15(\mathrm{~m}, 1 \mathrm{H}), 7.29-7.32(\mathrm{~m}, 1 \mathrm{H}), 7.52-7.57(\mathrm{~m}, 1 \mathrm{H}), 8.47-8.49(\mathrm{~m}$, $1 \mathrm{H}) ;{ }^{13} \mathrm{C}-\mathrm{NMR}: \delta 31.4(2 \mathrm{C}), 64.9,80.9,94.6,122.4,126.6,135.5,142.3,148.9$.

2-Methyl-4-(3-pyridyl)-3-butyn-2-ol (5j). Yellow solid. m.p. 55-56 ${ }^{\circ} \mathrm{C}$ (lit.[25] $\left.53{ }^{\circ} \mathrm{C}\right) .{ }^{1} \mathrm{H}-\mathrm{NMR}: \delta$ $1.58(\mathrm{~s}, 6 \mathrm{H}), 2.00(\mathrm{~s}, 1 \mathrm{H}), 7.18-7.22(\mathrm{~m}, 1 \mathrm{H}), 7.64-7.67(\mathrm{~m}, 1 \mathrm{H}), 8.44-8.45(\mathrm{~m}, 1 \mathrm{H}), 8.71(\mathrm{~s}, 1 \mathrm{H}) ;{ }^{13} \mathrm{C}-$ NMR: $\delta 31.6(2 \mathrm{C}), 64.9,78.1,98.3,120.0,122.8,138.4,147.3,151.3$.

3-Phenyl-2-propyn-1-ol (5k) [75]. Yellow oil. ${ }^{1} \mathrm{H}-\mathrm{NMR}: \delta 2.87(\mathrm{~s}, 1 \mathrm{H}), 4.50(\mathrm{~s}, 2 \mathrm{H}), 7.27-7.33$ (m, $3 \mathrm{H}), 7.42-7.45$ (m, 2H); ${ }^{13} \mathrm{C}-\mathrm{NMR}: \delta 51.4,85.3,87.1,123.1,127.8(2 \mathrm{C}), 127.9,131.1$ (2C).

3-(4'-Cyano)phenyl-2-propyn-1-ol (5l) [76]. Off-white solid. m.p. 89-91 ${ }^{\circ} \mathrm{C}$ (lit.[77] 87.5-88 $\left.{ }^{\circ} \mathrm{C}\right) .{ }^{1} \mathrm{H}-$ NMR: $\delta 2.01(\mathrm{~s}, 1 \mathrm{H}), 4.50(\mathrm{~s}, 2 \mathrm{H}), 7.48(\mathrm{~d}, J=6.4 \mathrm{~Hz}, 2 \mathrm{H}), 7.57(\mathrm{~d}, J=6.4 \mathrm{~Hz}, 2 \mathrm{H}) ;{ }^{13} \mathrm{C}-\mathrm{NMR}: \delta$ 51.6, 83.9, 91.6, 111.5, 118.0, 127.1, 131.5 (2C), 131.7 (2C).

4-(3-Hydroxy-1-propynyl)acetophenone (5m). Yellow solid. m.p. $80-81{ }^{\circ} \mathrm{C}$ (lit.[78] $\left.80-81{ }^{\circ} \mathrm{C}\right) .{ }^{1} \mathrm{H}-$ NMR: $\delta 2.55(\mathrm{~s}, 3 \mathrm{H}), 2.80(\mathrm{~s}, 1 \mathrm{H}), 4.90(\mathrm{~s}, 2 \mathrm{H}), 7.43(\mathrm{~d}, J=8.4 \mathrm{~Hz}, 2 \mathrm{H}), 7.82(\mathrm{~d}, J=8.4 \mathrm{~Hz}, 2 \mathrm{H}) ;{ }^{13} \mathrm{C}-$ NMR: $\delta 26.9,51.5,84.4,90.7,127.1,127.8(2 \mathrm{C}), 131.2(2 \mathrm{C}), 135.7,196.8$.

3-(4'-Nitro)phenyl-2-propyn-1-ol (5n). Yellow solid. m.p. 96-97 ${ }^{\circ} \mathrm{C}$ (lit.[79] 95-96.5 $\left.{ }^{\circ} \mathrm{C}\right) .{ }^{1} \mathrm{H}-\mathrm{NMR}: \delta$ $1.95(\mathrm{~s}, 1 \mathrm{H}), 4.52(\mathrm{~s}, 2 \mathrm{H}), 7.54(\mathrm{~d}, J=8.0 \mathrm{~Hz}, 2 \mathrm{H}), 8.15(\mathrm{~d}, J=8.0 \mathrm{~Hz}, 2 \mathrm{H}) ;{ }^{13} \mathrm{C}-\mathrm{NMR}: \delta 51.6,83.7$, 92.4, $123.2(2 \mathrm{C}), 129.0,131.9(2 \mathrm{C}), 146.6$.

3-(4'-Chlorophenyl)-2-propyn-1-ol (5o). Yellow solid. m.p. 77-79 ${ }^{\circ} \mathrm{C}$ (lit.[72] 78.5-79 $\left.{ }^{\circ} \mathrm{C}\right) .{ }^{1} \mathrm{H}-\mathrm{NMR}$ : $\delta 2.11(\mathrm{~s}, 1 \mathrm{H}), 4.47(\mathrm{~s}, 2 \mathrm{H}), 7.25(\mathrm{~d}, J=8.8 \mathrm{~Hz}, 2 \mathrm{H}), 7.33(\mathrm{~d}, J=8.8 \mathrm{~Hz}, 2 \mathrm{H}) ;{ }^{13} \mathrm{C}-\mathrm{NMR}: \delta 51.7,84.5$, $88.0,120.6,128.2(2 \mathrm{C}), 132.4(2 \mathrm{C}), 134.1$.

3-(2'-Thiophenyl)-2-propyn-1-ol (5p) [64]. Pale yellow oil. ${ }^{1} \mathrm{H}-\mathrm{NMR}: \delta 2.17(\mathrm{~s}, 1 \mathrm{H}), 4.50(\mathrm{~s}, 2 \mathrm{H})$, 6.95-6.97 (m, 1H), 7.20-7.21 (m, 1H), 7.25-7.26 (m, 1H); ${ }^{13} \mathrm{C}-\mathrm{NMR}: \delta$ 51.8, 78.8, 91.0, 122.0, 126.6, 127.0, 131.9 . 
3-(3'-Thiophenyl)-2-propyn-1-ol (5q) [54]. Brown oil. ${ }^{1} \mathrm{H}-\mathrm{NMR}: \delta 3.06$ (s, 1H), 4.46 (s, 2H), 7.06-7.08 (m, 1H), 7.19-7.24 (m, 1H), 7.41-7.42 (m, 1H); ${ }^{13} \mathrm{C}-\mathrm{NMR}: \delta$ 51.4, 80.6, 86.8, 121.1, 124.9, 128.6, 129.3 .

3-(2-Pyridyl)-2-propyn-1-ol (5r). White solid. m.p. 83-84 ${ }^{\circ} \mathrm{C}$ (lit.[80] $\left.82{ }^{\circ} \mathrm{C}\right) .{ }^{1} \mathrm{H}-\mathrm{NMR}: \delta 2.43(\mathrm{~s}, 1 \mathrm{H})$, $4.53(\mathrm{~s}, 2 \mathrm{H}), 7.19-7.22(\mathrm{~m}, 1 \mathrm{H}), 7.39-7.41(\mathrm{~m}, 1 \mathrm{H}), 7.60-7.64(\mathrm{~m}, 1 \mathrm{H}), 8.50-8.51(\mathrm{~m}, 1 \mathrm{H})$; ${ }^{13} \mathrm{C}-\mathrm{NMR}$ : $\delta 51.3,84.1,88.3,122.7,126.8,136.0,142.2,149.2$.

3-(3-Pyridyl)-2-propyn-1-ol (5s). White solid. m.p. 101-102 ${ }^{\circ} \mathrm{C}$ (lit.[81] 99-100 $\left.{ }^{\circ} \mathrm{C}\right) .{ }^{1} \mathrm{H}-\mathrm{NMR}: \delta 2.08$ $(\mathrm{s}, 1 \mathrm{H}), 4.49(\mathrm{~s}, 2 \mathrm{H}), 7.23-7.36(\mathrm{~m}, 1 \mathrm{H}), 7.70-7.74(\mathrm{~m}, 1 \mathrm{H}), 8.47-8.48(\mathrm{~m}, 1 \mathrm{H}), 8.74-8.75(\mathrm{~m}, 1 \mathrm{H})$; ${ }^{13} \mathrm{C}-\mathrm{NMR}: \delta$ 51.0, 81.3, 92.0, 119.9, 122.9, 138.5, 147.6, 151.4.

4-Phenyl-3-butyn-1-ol (5t) [82]. Light brown oil. ${ }^{1} \mathrm{H}-\mathrm{NMR}: \delta 1.84(\mathrm{t}, J=6.2 \mathrm{~Hz}, 1 \mathrm{H}), 2.70$ (t, $J=6.2 \mathrm{~Hz}, 2 \mathrm{H}), 3.82(\mathrm{q}, J=6.2 \mathrm{~Hz}, 2 \mathrm{H}), 7.26-7.32(\mathrm{~m}, 3 \mathrm{H}), 7.39-7.44(\mathrm{~m}, 2 \mathrm{H}) ;{ }^{13} \mathrm{C}-\mathrm{NMR}: \delta 23.5$, $60.9,82.1,86.5,123.3,127.7,128.1(2 \mathrm{C}), 131.5(2 \mathrm{C})$.

4-(4'-Methoxy)phenyl-3-butyn-1-ol (5u). Pale yellow solid. m.p. $58-59{ }^{\circ} \mathrm{C}$ (lit.[83] $\left.61{ }^{\circ} \mathrm{C}\right) .{ }^{1} \mathrm{H}-\mathrm{NMR}: \delta$ $1.90(\mathrm{br}, 1 \mathrm{H}), 2.65(\mathrm{t}, J=6.1 \mathrm{~Hz}, 2 \mathrm{H}), 3.77(\mathrm{t}, J=6.1 \mathrm{~Hz}, 2 \mathrm{H}), 6.80(\mathrm{~d}, J=8.6 \mathrm{~Hz}, 2 \mathrm{H}), 7.32(\mathrm{~d}$, $J=8.6 \mathrm{~Hz}, 2 \mathrm{H}) ;{ }^{13} \mathrm{C}-\mathrm{NMR}: \delta 23.6,55.1,61.1,81.9,84.8,113.7$ (2C), 115.4, 132.8 (2C), 159.1 .

4-(4'-Cyano)phenyl-3-butyn-1-ol (5v) [53]. Pale yellow solid. m.p. 80-81 ${ }^{\circ} \mathrm{C} .{ }^{1} \mathrm{H}-\mathrm{NMR}: \delta 1.97$ (br, $1 \mathrm{H}), 2.69(\mathrm{t}, J=6.2 \mathrm{~Hz}, 2 \mathrm{H}), 3.81(\mathrm{t}, J=6.2 \mathrm{~Hz}, 2 \mathrm{H}), 7.44(\mathrm{~d}, J=8.4 \mathrm{~Hz}, 2 \mathrm{H}), 7.54(\mathrm{~d}, J=8.4 \mathrm{~Hz}$, $2 \mathrm{H}) ;{ }^{13} \mathrm{C}-\mathrm{NMR}: \delta 23.6,60.6,80.6,91.8,110.8,118.3,128.4,131.7$ (2C), $132.0(2 \mathrm{C})$.

4-(4-Hydroxy-1-butynyl)acetophenone (5w). Pale yellow solid. m.p. $75-77{ }^{\circ} \mathrm{C}$ (lit.[53] 74-76 $\left.{ }^{\circ} \mathrm{C}\right) .{ }^{1} \mathrm{H}-$ NMR: $\delta 2.00(\mathrm{br}, 1 \mathrm{H}), 2.56(\mathrm{~s}, 3 \mathrm{H}), 2.69(\mathrm{t}, J=6.2 \mathrm{~Hz}, 2 \mathrm{H}), 3.81(\mathrm{t}, J=6.2 \mathrm{~Hz}, 2 \mathrm{H}), 7.45(\mathrm{~d}$, $J=8.2 \mathrm{~Hz}, 2 \mathrm{H}), 7.85(\mathrm{~d}, J=8.2 \mathrm{~Hz}, 2 \mathrm{H}) ;{ }^{13} \mathrm{C}-\mathrm{NMR}: \delta 23.7,26.4,60.8,81.4,90.5,128.0(2 \mathrm{C}), 128.4$, $131.6(2 \mathrm{C}), 135.7,197.4$.

4-(2-Thiophenyl)-3-butyn-1-ol (5x) [54]. Light brown oil. ${ }^{1} \mathrm{H}-\mathrm{NMR}: \delta 1.93$ (br, 1H), 2.69 (t, $J=6.2 \mathrm{~Hz}, 2 \mathrm{H}), 3.79(\mathrm{t}, J=6.2 \mathrm{~Hz}, 2 \mathrm{H}), 6.92(\mathrm{dd}, J=5.1,3.6 \mathrm{~Hz}, 1 \mathrm{H}), 7.14(\mathrm{~d}, J=3.6 \mathrm{~Hz}, 1 \mathrm{H}), 7.18$ $(\mathrm{d}, J=5.2 \mathrm{~Hz}, 1 \mathrm{H}) ;{ }^{13} \mathrm{C}-\mathrm{NMR}: \delta 24.0,60.9,75.4,90.5,123.3,126.2,126.7,131.4$.

4-(2-Pyridyl)-3-butyn-1-ol (5y) [25]. Light brown oil. ${ }^{1} \mathrm{H}$ NMR: $\delta 2.72$ (t, J=6.0 Hz, 2H), 3.06 (br, $1 \mathrm{H}), 3.87(\mathrm{t}, J=6.0 \mathrm{~Hz}, 2 \mathrm{H}), 7.18-7.25(\mathrm{~m}, 1 \mathrm{H}), 7.39(\mathrm{~d}, J=7.8 \mathrm{~Hz}, 1 \mathrm{H}), 7.64(\mathrm{td}, J=7.8,2.0 \mathrm{~Hz}$, $1 \mathrm{H}), 8.54(\mathrm{~d}, J=5.0 \mathrm{~Hz}, 1 \mathrm{H}) ;{ }^{13} \mathrm{C}-\mathrm{NMR}: \delta 23.3,59.9,80.7,88.4,122.2,126.4,136.0,142.7,148.8$.

\subsection{General procedure for recycling of nanosized MCM-41-Pd}

Under a nitrogen atmosphere, a $50 \mathrm{~mL}$ Schlenk tube was charged with NS-MCM-41-Pd (50 mg, $\left.7.5 \times 10^{-3} \mathrm{mmol}-\mathrm{Pd}\right), \mathrm{CuI}\left(2.9 \mathrm{mg}, 1.5 \times 10^{-2} \mathrm{mmol}\right), \mathrm{PPh}_{3}\left(3.9 \mathrm{mg}, 1.5 \times 10^{-2} \mathrm{mmol}^{2}, \mathrm{Et}_{3} \mathrm{~N}(15 \mathrm{~mL})\right.$, aryl halide $(7.5 \mathrm{mmol})$, and terminal alkyne $(8.3 \mathrm{mmol}$; in the case of $\mathbf{4 b}, 11.3 \mathrm{mmol}$ was used $)$. The 
mixture was stirred at $50{ }^{\circ} \mathrm{C}$ for $3 \mathrm{~h}(6 \mathrm{~h}$ for Entry 2$)$ and then cooled to room temperature. Recovery of NS-MCM-41-Pd was achieved by centrifugation and successive washes with THF, $\mathrm{H}_{2} \mathrm{O}$, and THF ( $2 \times 40 \mathrm{~mL}$ each washing). The solid was then dried under vacuum overnight and used for the next run.

\section{Conclusions}

In conclusion, NS-MCM-41-Pd is a highly efficient and recyclable catalyst for the coupling of a wide variety of aryl and heteroaryl halides with terminal alkynes, requiring catalyst loadings as low as $0.01 \mathrm{~mol} \%$ for a single run. The NS-MCM-41-Pd catalyst also exhibited excellent reusability when a catalyst loading of only $0.1 \mathrm{~mol} \%$ was employed for the recycling studies. The results of this study demonstrate the usefulness of anchored palladium bipyridyl complex on mesoporous silica as a heterogeneous catalyst in cross-coupling reactions.

\section{Acknowledgements}

This research was financially supported by the National Science Council of Taiwan (NSC95-2113M-027-001).

\section{References and Notes}

1. Sonogashira, K.; Tohda, Y.; Hagihara, N. A convenient synthesis of acetylenes: catalytic substitutions of acetylenic hydrogen with bromoalkenes, iodoarenes and bromopyridines. Tetrahedron Lett. 1975, 16, 4467-4470.

2. Moore, J.S. Shape-persistent molecular architectures of nanoscale dimension. Acc. Chem. Res. 1997, 30, 402-413.

3. Sonogashira, K. Metal-Catalyzed Cross-Coupling Reactions; Diederich, F., Stang, P.J., Eds.; Wiley-VCH: Weinheim, Germany, 1998; pp. 203-209.

4. Brandsma, L.; Vasilevsky, S.F.; Verkruijsse, H.D. Application of Transition Metal Catalysts in Organic Synthesis; Springer: Berlin, Germany, 1998; pp. 179-225.

5. Sonogashira, K. Handbook of Organopalladium Chemistry for Organic Synthesis; Negishi, E., de Meijere, A., Eds.; Wiley-VCH: New York, NY, USA, 2002; p. 493.

6. Sonogashira, K. Development of $\mathrm{Pd}-\mathrm{Cu}$ catalyzed cross-coupling of terminal acetylenes with $\mathrm{sp}^{2}$ carbon halides. J. Organomet. Chem. 2002, 653, 46-49.

7. Negishi, E.; Anastasia, L. Palladium-catalyzed alkynylation. Chem. Rev. 2003, 103, 1979-2017.

8. Taylor, E.C.; Dowling, J.E. Replacement of the 1',4'-phenylene region in 5,10-dideaza-5,6,7,8tetrahydrofolic acid (DDATHF) by 4,5,6,7-tetrahydrobenzo[c]thiophene and 4,5,6,7tetrahydroisobenzofuran Nuclei. J. Org. Chem. 1997, 62, 1599-1603.

9. Nakamura, H.; Aizawa, M.; Takeuchi, D.; Murai, A.; Shimoura, O. Convergent and short-step syntheses of dl-Cypridina luciferin and its analogues based on Pd-mediated cross couplings. Tetrahedron Lett. 2000, 41, 2185-2188.

10. Liu, T.-Z.; Isobe, M. Synthesis of the H-I-J tricyclic fragment of ciguatoxin, a marine polyether toxin. Synlett 2000, 266-268. 
11. de Kort, M.; Correa, V.; Valentijn, A.R.P.M.; van der Marel, G.A.; Potter, B.V.L.; Taylor, C.W.; van Boom, J.H. Synthesis of potent agonists of the D-myo-inositol 1,4,5-trisphosphate receptor based on clustered disaccharide polyphosphate analogues of adenophostin A. J. Med. Chem. 2000, 43, 3295-3303.

12. Amiet, G.; Hügel, H.M.; Nurlawis, F. The synthesis of the kynurenamines K1 and K2, metabolites of melatonin. Synlett 2002, 495-497.

13. Cosford, N.D.P.; Tehrani, L.; Roppe, J.; Schweiger, E.; Smith, N.D.; Anderson, J.; Bristow, L.; Brodkin, J.; Jiang, X.; McDonald, I.; Rao, S.; Washburn, M.; Varney, M.A. 3-[(2-Methyl-1,3thiazol-4-yl)ethynyl]-pyridine: A potent and highly selective metabotropic glutamate subtype 5 receptor antagonist with anxiolytic activity. J. Med. Chem. 2003, 46, 204-206.

14. Nicolaou, K.C.; Dai, W.-M. Chemistry and biology of the enediyne anticancer antibiotics. Angew. Chem., Int. Ed. Engl. 1991, 30, 1387-1416.

15. Yoshimura, F.; Kawata, S.; Hirama, M. Synthetic study of kedarcidin chromophore: Atropselective construction of the ansamacrolide. Tetrahedron Lett. 1999, 40, 8281-8285.

16. Toyota, M.; Komori, C.; Ihara, M. A concise formal total synthesis of mappicine and nothapodytine B via an intramolecular hetero Diels-Alder reaction. J. Org. Chem. 2000, 65, 7110-7113.

17. Paterson, I.; Davies, R.D.M.; Marquez, R. Total synthesis of the callipeltoside Aglycon. Angew. Chem., Int. Ed. 2001, 40, 603-607.

18. Kiehl, A.; Müllen, K. Catalysis in Precision Polymerization; Kobayashi, S., Eds.; Wiley-VCH: Chichester, England, 1997; pp. 162-169.

19. Bunz, U.H.F. Poly(aryleneethynylene)s: Syntheses, properties, structures, and applications. Chem. Rev. 2000, 100, 1605-1644.

20. Höger, S.; Rosselli, S.; Ramminger, A.-D.; Enkelmann, V. A facile synthesis of large extraannular-functionalized phenyl-ethynyl macrocycles containing m-terphenyl units. Org. Lett. 2002, 4, 4269-4272.

21. Li, C.-J.; Slaven, W.T., IV; John, V.T.; Banerjee, S. Palladium catalysed polymerization of aryl diodides with acetylene gas in aqueous medium: A novel synthesis of areneethynylene polymers and oligomers. Chem. Commun. 1997, 1569-1570.

22. Sonogashira, K. Comprehensive Organic Synthesis; Trost, B.M., Fleming, I., Eds.; Pergamon: New York, NY, USA, 1991; Volume 3, pp. 521-549.

23. Choudary, B.M.; Madhi, S.; Chowdari, N.S.; Kantam, M.L.; Sreedhar, B. Layered double hydroxide supported nanopalladium catalyst for Heck-, Suzuki-, Sonogashira-, and Stille-type coupling reactions of chloroarenes. J. Am. Chem. Soc. 2002, 124, 14127-14136.

24. Heidenreich, R.G.; Köhler, K.; Krauter, J.G.E.; Pietsch, J. Pd/C as a highly active catalyst for Heck, Suzuki and Sonogashira reactions. Synlett 2002, 1118-1122.

25. Novák, Z.; Szabó, A.; Répási, J.; Kotschy, A. Sonogashira coupling of aryl halides catalyzed by palladium on charcoal. J. Org. Chem. 2003, 68, 3327-3329.

26. Komáromi, A.; Novák, Z. Efficient copper-free Sonogashira coupling of aryl chlorides with palladium on charcoal. Chem. Commun. 2008, 4968-4970.

27. Mori, S.; Yanase, T.; Aoyagi, S.; Monguchi, Y.; Maegawa, T.; Sajiki, H. Ligand-free sonogashira coupling reactions with heterogeneous $\mathrm{Pd} / \mathrm{C}$ as the catalyst. Chem. Eur. J. 2008, 14, 6994-6999. 
28. Reddy, E.A.; Barange, D.K.; Islam, A.; Mukkanti, K.; Pal, M. Synthesis of 2-alkynylquinolines from 2-chloro and 2,4-dichloroquinoline via $\mathrm{Pd} / \mathrm{C}$-catalyzed coupling reaction in water. Tetrahedron 2008, 64, 7143-7150.

29. Shang, H.; Hua, R.; Zheng, Q.; Zhang, J.; Liang, X.; Zhu, Q. An improved practical Pd/Ccatalyzed Sonogashira cross-coupling reaction for the synthesis of liquid crystals of transcyclohexyltolans. Appl. Organomet. Chem. 2010, 24, 473-476.

30. Duplais, C.; Forman, A.J.; Baker, B.A.; Lipshutz, B.H. UC Pd: A new form of PdVC for sonogashira couplings. Chem. Eur. J. 2010, 16, 3366-3371.

31. Li, P.; Wang, L.; Li, H. Application of recoverable nanosized palladium(0) catalyst in Sonogashira reaction. Tetrahedron 2005, 61, 8633-8640.

32. Chouzier, S.; Gruber, M.; Djakovitch, L. New hetero-bimetallic Pd-Cu catalysts for the one-pot indole synthesis via the Sonogashira reaction. J. Mol. Catal. A: Chem. 2004, 212, 43-52.

33. Djakovitch, L.; Rollet, P. Sonogashira cross-coupling reactions catalysed by copper-free palladium zeolites. Adv. Synth. Catal. 2004, 346, 1782-1792.

34. Djakovitch, L.; Rollet, P. Sonogashira cross-coupling reactions catalysed by heterogeneous copper-free Pd-zeolites. Tetrahedron Lett. 2004, 45, 1367-1370.

35. Tyrrell, E.; Whiteman, L.; Williams, N. Sonogashira cross-coupling reactions and construction of the indole ring system using a robust, silica-supported palladium catalyst. Synthesis 2009, 829-835.

36. Anwander, R. SOMC@PMS. Surface organometallic chemistry at periodic mesoporous silica. Chem. Mater. 2001, 13, 4419-4438.

37. Biffis, A.; Zecca, M.; Basato, M. Palladium metal catalysts in Heck C-C coupling reactions. J. Mol. Catal. A: Chem. 2001, 173, 249-274.

38. He, X.; Antonelli, D. Recent advances in synthesis and applications of transition metal containing mesoporous molecular sieves. Angew. Chem. Int. Ed. 2002, 41, 215-229.

39. De Vos, D.E.; Dams, M.; Sels, B.F.; Jacobs, P.A. Ordered mesoporous and microporous molecular sieves functionalized with transition metal complexes as catalysts for selective organic transformations. Chem. Rev. 2002, 102, 3615-3640.

40. Trong On, D.; Desplantier-Giscard, D.; Danumah, C.; Kaliaguine, S. Perspectives in catalytic applications of mesostructured materials. Appl. Catal. A: Gen. 2003, 253, 545-602.

41. Thomas, J.M.; Raja, R. Catalytic significance of organometallic compounds immobilized on mesoporous silica: Economically and environmentally important examples. J. Organomet. Chem. 2004, 689, 4110-4124.

42. Li, C. Chiral synthesis on catalysts immobilized in microporous and mesoporous materials. Catal. Rev. 2004, 46, 419-492.

43. Taguchi, A.; Schüth, F. Ordered mesoporous materials in catalysis. Microporous Mesoporous Mater. 2005, 77, 1-45.

44. Hoffmann, F.; Cornelius, M.; Mprell, J.; Fröba, M. Silica-based mesoporous organic-inorganic hybrid materials. Angew. Chem., Int. Ed. 2006, 45, 3216-3251.

45. Rollet, P.; Kleist, W.; Dufaud, V.; Djakovitch, L. Copper-free heterogeneous catalysts for the Sonogashira cross-coupling reaction: Preparation, characterisation, activity and applications for organic synthesis. J. Mol. Catal. A: Chem. 2005, 241, 39-51. 
46. Cai, M.; Xu, Q.; Wang, P. A novel MCM-41-supported sulfur palladium(0) complex catalyst for Sonogashira coupling reaction. J. Mol. Catal. A: Chem. 2006, 250, 199-202.

47. Cai, M.; Sha, J.; Xu, Q. MCM-41-supported bidentate phosphine palladium(0) complex: a highly active and recyclable catalyst for the Sonogashira reaction of aryl iodides. Tetrahedron 2007, 63, 4642-4647.

48. Cai, M.; Xu, Q.; Sha, J. Copper-free Sonogashira coupling reaction catalyzed by MCM-41supported thioether palladium(0) complex in water under aerobic conditions. J. Mol. Catal. A: Chem. 2007, 272, 293-297.

49. Alonso, D.A.; Nájera, C.; Pacheco, M.C. A copper- and amine-free Sonogashira-type coupling procedure catalyzed by oxime palladacycles. Tetrahedron Lett. 2002, 43, 9365-9368.

50. McGuinness, D.S.; Cavell, K.J. Donor-Functionalized Heterocyclic Carbene Complexes of Palladium(II): Efficient Catalysts for C-C Coupling Reactions. Organometallics 2000, 19, 741-746.

51. Buchmeiser, M.R.; Schareina, T.; Kempe, R.; Wurst, K. Bis(pyrimidine)-based palladium catalysts: Synthesis, X-ray structure and applications in Heck-, Suzuki-, Sonogashira-Hagihara couplings and amination reactions. J. Organomet. Chem. 2001, 643, 39-46.

52. Feuerstein, M.; Berthiol, F.; Doucet, H.; Santelli, M. Palladium-tetraphosphine complex: An efficient catalyst for the coupling of aryl halides with alkynes. Org. Biomol. Chem. 2003, 1, 2235-2237.

53. Feuerstein, M.; Doucet, H.; Santelli, M. Coupling reactions of aryl bromides with 1-alkynols catalysed by a tetraphosphine/palladium catalyst. Tetrahedron Lett. 2004, 45, 1603-1606.

54. Feuerstein, M.; Doucet, H.; Santelli, M. Sonogashira cross-coupling reactions with heteroaryl halides in the presence of a tetraphosphine-palladium catalyst. Tetrahedron Lett. 2005, 46, $1717-1720$.

55. Tsai, F.-Y.; Wu, C.-L.; Mou, C.-Y.; Chao, M.-C.; Lin, H.-P.; Liu, S.-T. Palladium bipyridyl complex anchored on nanosized MCM-41 as a highly efficient and recyclable catalyst for Heck reaction. Tetrahedron Lett. 2004, 45, 7503-7506.

56. Tsai, F.-Y.; Lin, B.-N.; Chen, M.-J.; Mou, C.-Y.; Liu, S.-T. Anchored palladium bipyridyl complex in nanosized MCM-41: a recyclable and efficient catalyst for the Kumada-Corriu reaction. Tetrahedron 2007, 63, 4304-4309.

57. Chen, J.-Y.; Chen, S.-C.; Tang, Y.-J.; Mou, C.-Y.; Tsai, F.-Y. Coupling of acyl chlorides with triarylbismuths catalyzed by palladium bipyridyl complex anchored on nanosized MCM-41: A recyclable and atom-efficient catalytic process for the synthesis of diaryl and alkyl aryl ketones. $J$. Mol. Catal. A: Chem. 2009, 307, 88-92.

58. Chen, J.-Y.; Lin, T.-C.; Chen, S.-C.; Chen, A.-J.; Mou, C.-Y.; Tsai, F.-Y. Highly-efficient and recyclable nanosized MCM-41 anchored palladium bipyridyl complex-catalyzed coupling of acyl chlorides and terminal alkynes for the formation of ynones. Tetrahedron 2009, 65, 10134-10141.

59. Oki, A.R.; Morgan, R.J. An efficient preparation of 4,4'-dicarboxy-2,2'-bipyridine. Synth. Commun. 1995, 25, 4093-4097.

60. Will, G.; Boschloo, G.; Rao, S.N.; Fitzmaurice, D. Potentiostatic modulation of the lifetime of light-induced charge separation in a heterosupermolecule. J. Phys. Chem. B 1999, 103, 8067-8079. 
61. Lin, H.-P.; Tsai, C.-P. Synthesis of Mesoporous Silica Nanoparticles from a Low-concentration CnTMAX-Sodium Silicate Components. Chem. Lett. 2003, 32, 1092-1093.

62. Elangovan, A.; Wang, Y.-H.; Ho, T.-I. Sonogashira coupling reaction with diminished homocoupling. Org. Lett. 2003, 5, 1841-1844.

63. Kabalka, G.W.; Wang, L.; Pagni, R.M. Sonogashira coupling and cyclization reactions on alumina: A route to aryl alkynes, 2-substituted-benzo[b]furans and 2-substituted-indoles. Tetrahedron 2001, 57, 8017-8028.

64. Van den Hoven, B.G.; Alper, H. The first regioselective hydroformylation of acetylenic thiophenes catalyzed by a zwitterionic rhodium complex and triphenyl phosphate. J. Org. Chem. 1999, 64, 9640-9645.

65. Sørensen, U.S.; Pombo-Villar, E. Copper-free palladium-catalyzed sonogashira-type coupling of aryl halides and 1-aryl-2-(trimethylsilyl)acetylenes. Tetrahedron 2005, 61, 2697-2703.

66. Novák, Z.; Nemes, P.; Kotschy, A. Tandem Sonogashira coupling: An efficient tool for the synthesis of diarylalkynes. Org. Lett. 2004, 6, 4917-4920.

67. Pal, M.; Subramanian, V.; Parasuraman, K.; Yeleswarapu, K.R. Palladium catalyzed reaction in aqueous DMF: Synthesis of 3-alkynyl substituted flavones in the presence of prolinol. Tetrahedron 2003, 59, 9563-9570.

68. Kayaki, Y.; Yamamoto, M.; Ikariya, T. Stereoselective formation of $\alpha$-alkylidene cyclic carbonates via carboxylative cyclization of propargyl alcohols in supercritical carbon dioxide. $J$. Org. Chem. 2007, 72, 647-649.

69. Pourjavadi, A.; Marandi, G.B. Preparation of conjugated enynes and arylacetylenic compounds from arylalkynols using alumina in dry media. J. Chem. Res. 2002, 11, 552-555.

70. Adjabeng, G.; Brenstrum, T.; Frampton, C.S.; Robertson, A.J.; Hillhouse, J.; McNulty, J.; Capretta, A. Palladium complexes of 1,3,5,7-tetramethyl-2,4,8-trioxa-6-phenyl-6phosphaadamantane: Synthesis, crystal structure and use in the Suzuki and Sonogashira reactions and the $\alpha$-arylation of ketones. J. Org. Chem. 2004, 69, 5082-5086.

71. Kondo, K.; Fujitani, T.; Ohnishi, N. Synthesis and non-linear properties of disubstituted diphenylacetylene and related compounds. J. Mater. Chem. 1997, 7, 429-433.

72. Alonso, D.A.; Najera, C.; Pacheco, M.C. $\mathrm{C}\left(s p^{2}\right)-\mathrm{C}(s p)$ and $\mathrm{C}(s p)-\mathrm{C}(s p)$ coupling reactions catalyzed by oxime-derived palladacycles. Adv. Synth. Catal. 2003, 345, 1146-1158.

73. Sarkar, A.; Talwar, S.S. Heteroaryl functionalised diacetylenes: Preparation and solid-state reactivity. J. Chem. Soc., Perkin Trans. 1, 1998, 4141-4146.

74. Sarkar, A.; Manjunath, S.P.; Kamath, B.; Bhagwat, L.; Babu, K.N.; Rajalakshmi, K.; Talwar, S.S. A convenient method for the preparation of thienylacetylnes. Indian J. Chem. Sect. B, 1991, 30, 360-362.

75. Ishikawa, T.; Mizuta, T.; Hagiwara, K.; Aikawa, T.; Kudo, T.; Saito, S. Catalytic alkynylation of ketones and aldehydes using quaternary ammonium hydroxide base. J. Org. Chem. 2003, 68, 3702-3705.

76. Bernini, R.; Cacchi, S.; Fabrizi, G.; Forte, G.; Petrucci, F.; Prastaro, A.; Niembro, S.; Shafir A.; Vallribera, A. Alkynylation of aryl halides with perfluoro-tagged palladium nanoparticles immobilized on silica gel under aerobic, copper- and phosphine-free conditions in water. Org. Biomol. Chem. 2009, 7, 2270-2273. 
77. Bumagin, N.A.; Ponomaryov, A.B.; Beletskaya, I.P. A convenient synthesis of substituted propargyl alcohols and terminal acetylenes. Synthesis 1984, 9, 728-729.

78. Batey, R.A.; Shen, M.; Lough, A.J. Carbamoyl-substituted N-heterocyclic carbene complexes of palladium(II): Application to Sonogashira cross-coupling reactions. Org. Lett. 2002, 4, 1411-1414.

79. Harris, M.A.; McMillan, I.; Nayler, J.H.C.; Osborne, N.F.; Pearson, M.J.; Southgate, R. Syntheses based on 1,2-secopenicillins. Part II. Preparation of 4-(3-substituted prop-2-ynylthio)azetidin-2ones. J. Chem. Soc., Perkin Trans. 1, 1976, 1612-1615.

80. Al-Arnaout, A.; Courtois, G.; Miginiac, L. Synthèse régiosélective par voie organométallique de pyridines, 4-picolines et 3,5-lutidines substituées en 2 par un groupe insaturé et/ou fonctionnel. J. Organomet. Chem. 1987, 333, 139-154.

81. Bleicher, L.S.; Cosford, N.D.P.; Herbaut, A.; McCallum, J.S.; McDonald, I.A. A practical and efficient synthesis of the selective neuronal acetylcholine-gated ion channel agonist (S)-(-)-5ethynyl-3-(1-methyl-2-pyrrolidinyl)pyridine maleate (SIB-1508Y). J. Org. Chem. 1998, 63, $1109-1118$.

82. Kim, I.S.; Dong, G.R.; Jung, Y.H. Palladium(II)-catalyzed isomerization of olefins with tributyltin hydride. J. Org. Chem. 2007, 72, 5424-5426.

83. Collins, C.J.; Hanack, M.; Stutz, H.; Auchter, G.; Schoberth, W. Vinyl cations. 41. Influence of 4aryl and 4-alkyl substituents on the $\pi$-route solvolyses of homopropargyl esters. J. Org. Chem. 1983, 48, 5260-5268.

Sample Availability: Samples of the compounds are available from the authors.

(C) 2010 by the authors; licensee MDPI, Basel, Switzerland. This article is an open access article distributed under the terms and conditions of the Creative Commons Attribution license (http://creativecommons.org/licenses/by/3.0/). 